\title{
THE METHOD ESTIMATING IQ IMBALANCE USING SIGNAL POWER CONSTRAINED CONDITION IN OFDM SYSTEM
}

\author{
Yong Chan Lee ${ }^{1}$, Song Chol Ho ${ }^{2}$, Won Chol Jang ${ }^{3}$ \\ ${ }^{1,2}$ College of Information Engineering, Wonsan Jogunsil University of Technology, D.P.R. of Korea \\ ${ }^{3}$ Institute of Information Science, Kim Il Sung University, D.P.R. of Korea
}

\begin{abstract}
IQI (I/Q Imbalance) is caused by I, $Q$ path gain disagreement and quadrature phase incomplete generation $\triangle \varphi$. IQI is parasitic noise about one frequency in LO frequency band. IQI estimation and compensating method analyzed on slow frequency fading channel and all most previous works estimate and compensate FIIQI only and it has much complexity, as adaptive and Kalman filtering is done when estimating and compensating FSIQI. We propose the method estimating and compensating method using signal energy constrained condition. In this method, FI and FS IQI are compensated simultaneously and Kalman filtering is not used. Also, the robustness to frequency selective channel is high.
\end{abstract}

\section{Keywords:}

OFDM, IQ Imbalance, Frequency Fading Channel

\section{INTRODUCTION}

IQI is caused by following factors [1], [2]. First, because the phases of IQ local oscillator (LO) is different from ideal $90^{\circ}$. Second, because the amplifier coefficients of IQ Los are different. Most of IQI caused by above two causes and these two causes are called IQ amplitude and phase mismatching [1], [2]. Third, because of mismatching of amplifier coefficients and cut off frequencies of IQ filters. IQI is divided into FI (Frequency Independent) IQI and FS (Frequency Selective) IQI whether IQI is varied according to frequency. Mixer mismatching is FIIQI and filter mismatching is FSIQI. Usually mixer mismatching is researched and nth received symbol of $l^{\text {th }}$ OFDM symbol affected by IQI for amplitude imbalance $\varepsilon$ and phase imbalance $\Delta \phi$ is as follows.

$$
\begin{gathered}
y_{l}(n)=u r_{l}(n)+v r_{l}^{-x}(n)+w_{l}(n) \\
r_{l}=h_{l}(n) \otimes x_{l}(n)
\end{gathered}
$$

where, $x_{l}(n)$ is time domain complex signal transmitted after IFFT, $w_{l}$ is AWGN (Additive Whiten Gaussian Noise) and $h_{l}(n)$ is CIR (Channel Impulse Response) and $u$ and $v$ are IQI parameters.

$$
u=\frac{1+\varepsilon \exp (-j \Delta \varphi)}{2}, v=\frac{1-\varepsilon \exp (j \Delta \varphi)}{2}
$$

Received OFDM symbol in frequency domain after IFFT Eq.(1) is as follows.

$$
Y_{l}(-k)=u H_{l}(n) \cdot x_{l}(k)+v H_{l}^{-x}(n-k) \cdot x_{l}^{-x}(n-k)+w_{l}(k)(3)
$$

The Eq.(3) shows that IQI represents mirror symmetric subcarrier interference on the sub-carrier of original signal. By some experiment results, this ICI causes 5 9dB IL for BER $=10^{-3}$.

The IQI is usually estimated and compensated by using training sequence, or header and pilot symbols. In most of previous works, RXIQI, FSIQI and TXIQI are estimated and compensated all through.

The FS and FI TX and RX IQI are estimated and compensated in MIMO OFDM system as following.

The largest part of IQI is FIIQI in multi-antenna OFDM system that has $N_{t}$ transmitter and $N_{r}$ receivers and is caused in up-convert and down-convert mixers.

$N_{r} \times 1$ received vector $X_{m, k}$ of $k^{\text {th }}$ sub-carrier of $m^{\text {th }}$ symbol is as follows:

$$
\left(\begin{array}{l}
K_{1} \cdot H_{k} \cdot G_{1}+ \\
K_{2} \cdot H_{k}^{*} \cdot G_{2}
\end{array}\right) \cdot S_{m, k}+\left(\begin{array}{l}
K_{2} \cdot H_{k} \cdot G_{1}^{*}+ \\
K_{1} \cdot H_{k} \cdot G_{2}^{*}
\end{array}\right) \cdot S_{m, k}
$$

where $H_{k}: N_{r} \times N_{t}$ quasi channel transfer matrix and $S_{m, k}: N_{t} \times 1$ transmit signal vector.

In Eq.(4), TXIQI consists of $N_{t} \times N_{r}$ matrix $G_{1}$ and $G_{2}$, RXIQI consists of $N_{r} \times N_{r}$ matrix $K_{1}$ and $K_{2}$.

$$
\begin{cases}G_{1}=\frac{\left(I+g_{T} \cdot e^{j \varphi T}\right)}{2} & G_{2}=1-G_{1}^{*} \\ K_{1}=\frac{\left(I+g_{R} \cdot e^{j \varphi T}\right)}{2} & K_{2}=1-K_{1}^{*}\end{cases}
$$

Imbalance matrix is as follows.

$$
\begin{aligned}
& \varphi_{x}=\operatorname{diag}\left\{\varphi_{x, 1}, \varphi_{x, 2}, \ldots, \varphi_{x, N_{x}}\right\} \\
& g_{x}=\operatorname{diag}\left\{g_{x, 1}, g_{x, 2}, \ldots, g_{x, N_{x}}\right\}
\end{aligned}
$$

where $x \in\{T, R\} N_{x} \in\left\{N_{T}, N_{R}\right\}, g_{x, n}, \varphi_{x, n}$ is amplitude and phase imbalance in $n^{\text {th }}$ branch, if there is no demodulation, they are 0 and 1 , respectively.

If there is only RXIQI, $G_{1}=1 G_{2}=0$, if there is only TXIQI, $K_{1}=1, K_{2}=0$. FSIQI is caused by filter mismatching and delay difference in I, Q paths. FSIQI is modeled by only mismatching between receiver LPFs, as it is same as in receiver. Received signal in $k^{\text {th }}$ sub-carrier due to LPF mismatching is as follows.

$$
X_{m, k}=\frac{r_{l, k}+r_{\theta, k}}{2} \cdot H_{k} \cdot S_{m, k}+\frac{r_{l,-k}-r_{\theta,-k}}{2} \cdot H_{-k}^{*} \cdot S_{m,-k}^{*}
$$

where, $N_{r} \times N_{r}$ is the diagonal matrix and $r_{l, k}, r_{\theta, k}$ are $k^{\text {th }}$ sub-carrier frequency response in I, Q branch, respectively.

In special case that there is no imbalance, there is no extraction to original signal of display frequency, $r_{l, k}=r_{\theta, k}$. In practical system, the signal is represented by combination of FSIQI and FIIQI.

$$
\begin{aligned}
x_{m, k}= & \left(\hat{K}_{1, k} \cdot \hat{H}_{k} \cdot G_{1}+\hat{K}_{2, k} \cdot \hat{H}_{k}^{*} \cdot G_{2}\right) \cdot S_{m, k} \\
& +\left(\hat{K}_{2,-k} \cdot \hat{H}_{-k}^{*} \cdot G_{1}+\hat{K}_{1, k} \cdot \hat{H}_{k} \cdot G_{2}^{*}\right) \cdot S_{m,-k}^{*}
\end{aligned}
$$


where

$$
\begin{gathered}
\hat{K}_{1, k}=0.5\left(I+r_{I, k}^{-1} \cdot r_{\theta, k} \cdot g_{r} \cdot e^{-j \varphi R}\right) \\
\hat{K}_{2, k}=I-\hat{K}_{1, k}^{*} \\
\hat{H}_{k}=r_{I, k} \cdot H_{k}
\end{gathered}
$$

As $r_{I}$ and $r_{\theta}$ are frequency response of real filters, $r_{I, k}=r_{I, k}^{*}$, $r_{\theta, k}=r_{\theta,-k}^{*}$

\section{PROPOSED METHOD}

\subsection{ESTIMATION OF FIIQI}

The synchronization system is the one which enables the change of one side database to be made simultaneously at the other side database.

In general, the mathematical model of OFDM IQI is divided into two models.

The first model is as follows [3].

$$
\hat{Y}_{\ell}[k]=\gamma Y_{\ell}[k]+\lambda Y_{\ell}^{*}[-k]
$$

where

$$
\begin{aligned}
& \gamma=0.5\{1+(1+\varepsilon)(\cos \theta-j \sin \theta)\}, \\
& \lambda=0.5\{1-(1+\varepsilon)(\cos \theta+j \sin \theta)\}
\end{aligned}
$$

The second model is as follows [4].

$$
\hat{Y}_{\ell}[k]=\gamma Y_{\ell}[k]+\lambda Y_{\ell}^{*}[-k]
$$

where,

$$
\begin{aligned}
& \gamma=\cos \theta+j \varepsilon \sin \theta, \\
& \lambda=\varepsilon \cos \theta-j \sin \theta .
\end{aligned}
$$

In above equations, $\hat{Y}_{\ell}[k], Y_{\ell}[k]$ is the received signal in kth sub-carrier of $l^{\text {th }}$ OFDM symbol in case that was distorted by IQI and in case that there is no IQI. $\gamma$ and $\lambda$ are IQI parameters. $\varepsilon$ and $\theta$ are amplitude and phase imbalance parameters. In some previous works, AWGN $W_{l}[k]$ is added to Eq.(9) and Eq.(10).

IQI is usually estimated and compensated by training sequence and pilot symbols, but sometimes direct decision method is used [5].

In direct decision method, following boundary conditions are used.

- First, imbalance does not affect to the energy of signal.

- Second, I/Q components are both affected by identical imbalance.

These boundary conditions are satisfied in Eq.(10) and in Eq.(9) accurately too. The mathematical model of IQI in this paper is based on Eq.(10) and Eq.(11).

$$
\hat{Y}_{\ell}[k]=\gamma Y_{\ell}[k]+\lambda Y_{\ell}^{*}[-k]+W_{l}[k]
$$

where,

$$
\gamma=0.5\{1+(1+\varepsilon)(\cos \theta-j \sin \theta)\}
$$

$$
\begin{gathered}
\lambda=0.5\{1-(1+\varepsilon)(\cos \theta+j \sin \theta)\} \\
\gamma=\cos \theta+j \varepsilon \sin \theta \text { and } \\
\lambda=\varepsilon \cos \theta-j \sin \theta
\end{gathered}
$$

Considering that $Y_{l}[k]=X_{l}[k] H_{l}[k]$, then the expected value of square of absolute value is as follows.

$$
\begin{aligned}
& E\left[\left|\hat{Y}_{l}[k]\right|^{2}\right]=E\left[\gamma Y_{l}[k]+\lambda Y_{l}^{*}[-k]+W_{l}[k]^{2}\right] \\
= & E\left[\gamma X_{l}[k] H_{l}[k]+\lambda X_{l}^{*}[-k] H_{l}^{*}[-k]+W_{l}[k]^{2}\right] \\
= & E\left[\begin{array}{l}
\left(\gamma X_{l}[k] H_{l}[k]+\lambda X_{l}[-k] H_{l}[-k]+W_{l}[k]^{2}\right) \cdot \\
\left(\gamma X_{l}[k] H_{l}[k]+\lambda X_{l}^{*}[-k] H_{l}^{*}[-k]+W_{l}^{*}[k]^{2}\right)
\end{array}\right] \\
= & E\left[\begin{array}{l}
\left.|| \gamma\right|^{2} X_{l}[k] H_{l}[k]+\left.|\lambda|^{2} X_{l}[-k] H_{l}[-k]\right|^{2} \\
+2 \operatorname{Re}\left\{\gamma^{*} \lambda_{l}^{*}[k] Y_{l}^{*}[-k]\right\} \\
+2 \operatorname{Re}\left\{\left(\gamma Y_{l}[k]+\lambda Y_{l}^{*}[-k]\right)^{*} W_{l}[k]\right\}+W_{l}[k]^{2}
\end{array}\right]
\end{aligned}
$$

where $X_{l}[k], H_{l}[k]$ is a transmitted signal and CFR (Channel Frequency Response).

The fourth term of expected value of right hand of Eq.(13) is a product of signal and noise, its expected value is 0 . Also the third term is a product of received signals at each other subcarrier, its expected value is 0 too. Considering this, Eq.(13) is as follows.

$$
E\left[\left|\hat{Y}_{l}[k]\right|^{2}\right]=E\left[\begin{array}{l}
|\gamma|^{2}\left|X_{l}[k] H_{l}[k]\right|^{2} \\
+|\lambda|^{2}\left|X_{l}[-k] H_{l}[-k]\right|^{2} \\
+\left|W_{l}[k]\right|^{2}
\end{array}\right]
$$

In case that there is no IQI, that is, $\varepsilon=0$ and $\theta=0$, Eq.(14) is as follows.

$$
\hat{Y}_{l}^{0}[k]=Y_{l}[k]+W_{l}[k]=X_{l}[k] H_{l}[k]+W_{l}[k]
$$

From boundary condition 1 ,

$$
E\left[\left|\hat{Y}_{l}^{0}[k]\right|^{2}\right]=E\left[\left|\hat{Y}_{l}[k]\right|^{2}\right]
$$

$E\left[\left|\hat{Y}_{l}^{0}[k]\right|^{2}\right]=E\left[\left(X_{l}[k] H_{l}[k]+W_{l}[k]\right)\left(X_{l}[k] H_{l}[k]+W_{l}^{*}[k]\right)\right]$

$=E\left[\left|X_{l}[k] H_{l}[k]\right|^{2}+2 \operatorname{Re}\left\{W_{l}^{*}[k]\left(X_{l}[k] H_{l}[k]\right)\right\}+\left|W_{l}[k]\right|^{2}\right]$

$$
=E\left[\left|X_{l}[k] H_{l}[k]\right|^{2}\right]+E\left[\left|W_{l}[k]\right|^{2}\right]
$$

From Eq.(15) Eq.(17),

$$
E\left[\left|\begin{array}{l}
X_{l}[k] \\
H_{l}[k]
\end{array}\right|^{2}\right]=|\gamma|^{2} E\left[\left|\begin{array}{l}
X_{l}[k] \\
H_{l}[k]
\end{array}\right|^{2}\right]+|\lambda|^{2} E\left[\left|\begin{array}{c}
X_{l}[-k] \\
H_{l}[-k]
\end{array}\right|^{2}\right]
$$

From Eq.(17) and Eq.(18),

$$
|\gamma|^{2}+|\lambda|^{2}=1+\varepsilon^{2}
$$


Inserting Eq.(19) into Eq.(18),

$$
\begin{aligned}
& E\left[\left|Y_{l}[k]\right|^{2}\right]=|\gamma|^{2} E\left[\left|Y_{l}[k]\right|^{2}\right]+\left(1+\varepsilon^{2}-|\gamma|^{2}\right) E\left[\left|Y_{l}[-k]\right|^{2}\right] \\
& \Leftrightarrow\left(1-|\gamma|^{2}\right) E\left[\left|Y_{l}[k]\right|^{2}\right]=\left(1+\varepsilon^{2}-|\gamma|^{2}\right) E\left[\left|Y_{l}[-k]\right|^{2}\right] \\
& \Leftrightarrow \frac{E\left[\left|Y_{l}[k]\right|^{2}\right]}{E\left[\left|Y_{l}[-k]\right|^{2}\right]} \\
& =\frac{1+\varepsilon^{2}-|\gamma|^{2}}{1-|\gamma|^{2}} \\
& =1+\frac{\varepsilon^{2}}{1-\cos ^{2} \theta+\varepsilon^{2} \sin ^{2} \theta} \\
& =1+\frac{\varepsilon^{2}}{\sin ^{2} \theta+\varepsilon^{2} \sin ^{2} \theta} \\
& K_{-}^{+} \equiv \frac{E\left[\left|Y_{l}[k]\right|^{2}\right]}{E\left[\left|Y_{l}[-k]\right|^{2}\right]} \\
& K_{-}^{+}=1+\frac{\varepsilon^{2}}{\sin ^{2} \theta+\varepsilon^{2} \sin \theta} \Leftrightarrow\left(K_{-}^{+}-1\right)\left(\sin ^{2} \theta+\varepsilon^{2} \sin \theta\right) \\
& =\varepsilon^{2}\left(K_{-}^{+}-1\right) \sin ^{2} \theta \\
& =\varepsilon^{2}\left(1-\left(K_{-}^{+}-1\right) \sin ^{2} \theta\right) \frac{\left(K_{-}^{+}-1\right) \sin ^{2} \theta}{1-\left(K_{-}^{+}-1\right) \sin ^{2} \theta}=\varepsilon^{2}
\end{aligned}
$$

Inserting Eq.(23) into Eq.(11),

$$
\begin{gathered}
\hat{Y}_{l}[k]=\gamma Y_{l}[k]+\lambda Y_{l}^{*}[-K] \\
=\left(\sqrt{1-\theta^{2}}+j \sqrt{\frac{\left(K_{-}^{+}-1\right) \theta^{2}}{1-\left(K_{-}^{+}-1\right) \theta^{2}}}\right) X_{l}[k] H_{l}[k] \\
+\left(\sqrt{\left.\frac{\left(K_{-}^{+}-1\right) \theta^{2}}{1-\left(K_{-}^{+}-1\right) \theta^{2}} \sqrt{1-\theta^{2}}-j \theta\right) X_{l}[-k] H_{l}[-k]}\right.
\end{gathered}
$$

Consequently, the Eq.(24) is written with respect to one unknown quantity $\theta$.

If $X_{l}[k], X_{l}[-k], H_{l}[k]$ and $H_{l}[-k]$ are known, Eq.(24) can be solved easily. We denote $\theta$ obtained by solving Eq.(24) to $\hat{\theta}_{l}[k]$. We never used any approximate expressions to obtain $\hat{\theta}_{l}[k]$. After all, $\hat{\theta}_{l}[k]$ is the LS estimation of phase imbalance in $k^{\text {th }}$ sub-carrier of $l^{\text {th }}$ OFDM symbol and non-biased.

The amplitude imbalance parameter $\hat{\varepsilon}_{l}[k]$ is obtained by using Eq.(23) from $\hat{\theta}_{l}[k]$, where, $\hat{\theta}_{l}[k]$ is a constant with respect to $l$, as FIIQI parameters are independent to frequency. After all, $N_{p}$ $\hat{\varepsilon}_{l}$ and $\hat{\theta}_{l}$ are obtained by estimation of $\varepsilon_{l}$ and $\theta_{l}$, respectively.

Considering $W_{l}[k]$ in Eq.(24), as Eq.(24) is approximately linear with respect to $\hat{\theta}_{l}$, estimation accuracy of $\hat{\theta}_{l}$ is proportional to SNR in $k^{\text {th }}$ sub-carrier.

Obtaining real $\hat{\theta}_{l}$ from this measurement by weighted arithmetic average with weights SNR in $i^{\text {th }}$ pilot sub-carriers, $\Gamma_{i}^{l}$ is optimal in sense of MMSE than by arithmetic average [6]. That is, we obtain the phase imbalance of $l^{\text {th }}$ OFDM symbol by Eq.(25).

$$
\hat{\theta}_{l}=\frac{\sum_{i=1}^{N p} \Gamma_{i}^{l} \theta_{l}[i]}{\sum_{i=1}^{N p} \Gamma_{i}^{l}}
$$

where, $\hat{\varepsilon}_{l}$ is obtained by following equation just as before.

$$
\hat{\varepsilon}_{l}=\frac{\sum_{i=1}^{N p} \Gamma_{i}^{l} \varepsilon_{l}[i]}{\sum_{i=1}^{N p} \Gamma_{i}^{l}}
$$

\subsection{ESTIMATION OF FSIQI}

In FIIQI, $\varepsilon_{l}$ and $\theta_{l}$ are independent of sub-carrier, but in FSIQI, it is varied according to sub-carrier. So, $\hat{\theta}_{l}[k]$ and $\hat{\varepsilon}_{l}[k]$ obtained by Eq.(24) are estimation of parameters of FIIQI and FSIQI. However, $\hat{\theta}_{l}[k]$ and $\hat{\varepsilon}_{l}[k]$ are affected by noise $W_{l}[k]$ severely, if they are accepted as estimation of FIIQI and FSIQI in this case.

In order to overcome this, we have to take a weighted arithmetic average of estimations of IQI parameters in neighbor pilot sub-carriers, we cannot estimate FSIQI correctly.

We estimate $\hat{\theta}_{l}[k], \hat{\varepsilon}_{l}[k]$ to compromise mutual affection from these factors as following.

$$
\hat{\theta}_{l}[k]=\frac{\sum_{i=-m}^{m} \Gamma_{k+i \cdot d p}^{l} \theta_{l}[k+i \cdot d p] \cdot \gamma_{i}}{\sum_{i=-m}^{m} \Gamma_{k+i \cdot d p}^{l} \cdot \gamma_{i}}
$$

where, $d p$ is pilot sub-carrier spacing and $m$ is determined according to estimation of CIR change and the size of $W_{l}[k], 1 \sim 3$, and $\hat{\varepsilon}_{l}[k]$ is obtained by Eq.(28) just as before.

$$
\hat{\varepsilon}_{l}[k]=\frac{\sum_{i=-m}^{m} \Gamma_{k+i \cdot d p}^{l} \varepsilon_{l}[k+i \cdot d p]}{\sum_{i=-m}^{m} \Gamma_{k+i \cdot d p}^{l}}
$$

where $\gamma_{i}$ is set between 0.5 and 0.95 and depends on frequency change characteristics of SNR and FS characteristics of IQI. 


\subsection{INTERPOLATION OF IQI PARAMETERS}

As in the interpolation of FE parameters such as EFO/RFO and PHN, the distance interpolation filter is used in the interpolation of IQI parameters.

The interpolation of IQI parameters using the distance interpolation filter in general pilot pattern is as follows.

$$
\begin{aligned}
& \hat{\theta}(p d)=K \sum_{P p \in S c} \frac{\hat{\theta}(P p)}{d(P d, P p)} \\
& \hat{\varepsilon}(p d)=K \sum_{P p \in S c} \frac{\hat{\varepsilon}(P p)}{d(P d, P p)}
\end{aligned}
$$

where $\hat{\theta}(p d), \hat{\theta}(P p), \hat{\varepsilon}(p d), \hat{\varepsilon}(P p)$ is the phase and amplitude imbalance parameter estimation in data symbols and pilot symbols, respectively.

The density of $S_{c}$ using in Eq.(29) and Eq.(30) has to be set smaller than in estimation of PHN and CFO/RFO under identical condition, as change characteristics of IQI is almost similar to CIR. Of course, definite value depends on SNR in sub-carriers and IQI change.

\section{THE REALIZATION OF THE DATA SYNCHRONIZATION BASED ON THE WEB SERVICE}

The Fig.1 shows BER vs. SNR after removing the effects of IQI by using various IQI estimation and compensation methods. The proposed method has the smallest BER under condition that SNR is fixed compared with previous methods. This means that the proposed method removes IQI better than previous methods. For above 25dB SNR, 3 5dB BER decreases.

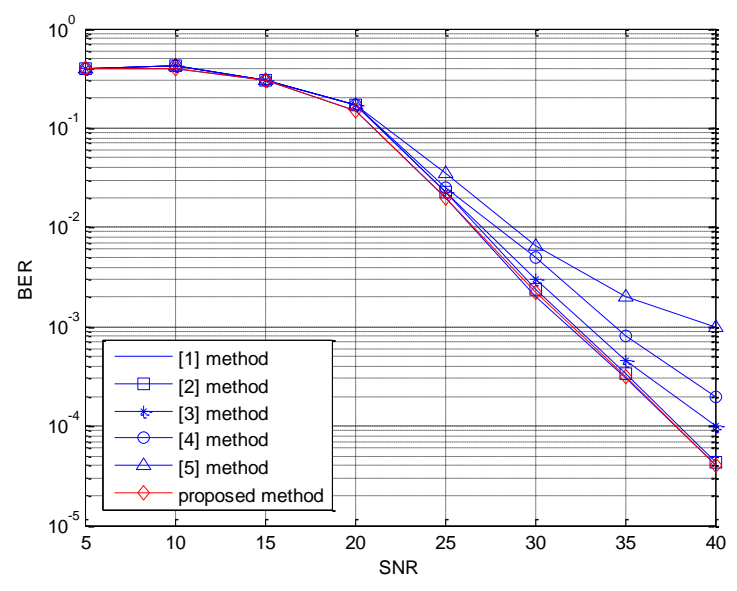

Fig.1. BER versus SNR after removing effects of IQI, for $\varepsilon=0.1, \theta=10^{\circ}$

\section{CONCLUSIONS}

We propose the method estimating and compensating method using signal energy constrained condition. In this method, FI and FSIQI are compensated simultaneously and Kalman filtering is not used. Also, the robustness to frequency selective channel is high. The proposed method has the smallest BER under condition that SNR is fixed compared with previous methods.

\section{REFERENCES}

[1] Tao Liu, "Joint Blind Estimation of Symbol Timing Offset and Carrier Frequency Offset for OFDM Systems with IQ Imbalance", IEICE Electronics Express, Vol. 16, No. 8, pp. 443-448, 2011.

[2] Lopez Estrviz, "Optimal Training Sequences for Joint Channel and Frequency-Dependent IQ Imbalance Estimation in OFDM", Proceedings of IEEE International Conference on Communications, pp. 11-15, 2006.

[3] J. Tubbax, "Compensation of IQ Imbalance and Phase Noise in OFDM systems", IEEE Transactions on Wireless Communications, Vol. 4, No. 3, pp. 872-877, 2005.

[4] Qiyue Zou, "Joint Compensation of IQ Imbalance and Phase Noise in OFDM Systems", IEEE Transactions on Wireless Communications, Vol. 57, No. 2, pp. 404-414, 2009.

[5] Qiyue Zou, "On the Joint Compensation of IQ Imbalances and Phase Noise in MIMO-OFDM Systems", Proceedings of IEEE International Symposium on Circuits and Systems, pp. 1-6, 2007. 